\title{
Structure-based Discovery of a new Protein-Aggregation Breaking Excipient
}

\author{
Tosstorff, Andreas; Svilenov, Hristo; Peters, Günther H.J.; Harris, Pernille; Winter, Gerhard
}

Published in:

European Journal of Pharmaceutics and Biopharmaceutics

Link to article, DOI:

10.1016/j.ejpb.2019.09.010

Publication date:

2019

Document Version

Peer reviewed version

Link back to DTU Orbit

Citation (APA):

Tosstorff, A., Svilenov, H., Peters, G. H. J., Harris, P., \& Winter, G. (2019). Structure-based Discovery of a new Protein-Aggregation Breaking Excipient. European Journal of Pharmaceutics and Biopharmaceutics, 144, 207216. https://doi.org/10.1016/j.ejpb.2019.09.010

\section{General rights}

Copyright and moral rights for the publications made accessible in the public portal are retained by the authors and/or other copyright owners and it is a condition of accessing publications that users recognise and abide by the legal requirements associated with these rights.

- Users may download and print one copy of any publication from the public portal for the purpose of private study or research.

- You may not further distribute the material or use it for any profit-making activity or commercial gain

- You may freely distribute the URL identifying the publication in the public portal 


\section{Structure-based Discovery of a new Protein-Aggregation Breaking Excipient}

Andreas Tosstorff ${ }^{1 *}$, Hristo Svilenov ${ }^{1}$, Günther H.J. Peters ${ }^{2}$, Pernille Harris ${ }^{2}$, Gerhard Winter ${ }^{1}$

${ }^{1}$ Department of Pharmacy, Pharmaceutical Technology and Biopharmaceutics, Ludwig-Maximilians-

Universität München, Munich, Germany

${ }^{2}$ Department of Chemistry, Technical University of Denmark, 2800 Kgs. Lyngby, Denmark

*Contact: andreas.tosstorff@cup.uni-muenchen.de 


\section{Abstract}

Reducing the aggregation of proteins is of utmost interest to the pharmaceutical industry. Aggregated proteins are often less active and can cause severe immune reactions in the patient upon administration. At the same time the biopharmaceutical market is pushing for high concentration formulations and products that do not require refrigerated storage conditions. For a given protein, the liquid formulation developer's toolbox is limited to achieve these goals: $\mathrm{pH}$, ionic strength and concentration of a very limited number of excipients are the only solution parameters to be varied. In this work, we present a structure-based approach to discover new molecules that successfully reduce the aggregation of proteins and apply it to the model protein Interferon-alpha-2a.

\section{Keywords}

Interferon-alpha-2a, Virtual Screen, Excipient, Protein Aggregation, Protein Formulation, Drug Discovery

\section{Introduction}

\section{Protein aggregation}

Protein aggregation is a major concern to regulatory agencies across the world. Not only can aggregation cause a decrease in biological activity, but the resulting aggregates have also been raising serious safety concerns as they can induce immunogenic side reactions upon parenteral injection(1). Pharmaceutical companies therefore strive to inhibit the formation of protein aggregates early on during drug development(2). Diagnostic proteins are another potential target to be stabilized by novel excipients.

The process of protein aggregation is very complex, with thermodynamics and kinetics depending on formulation conditions, stress, protein sequence and structure(3). Depending on the mechanism of aggregation, the resulting aggregates can consist of native or (partially) unfolded protein molecules. As shown by mutation experiments, hydrophobic patches on the proteins surface, so called 
aggregation hot-spots, are crucial to the formation of protein-protein interfaces, a key step in the formation of aggregates(4). There is a long list of available computational tools to identify aggregation hot-spots from a protein's primary sequence(5-7). Aggrescan3D (A3D) additionally takes into account the tertiary structural information of the protein, mitigating the risk of false positive results from hydrophobic residues buried within the protein fold(8).

\section{$\underline{\text { Excipients }}$}

Excipients reduce protein aggregation by various mechanisms of action. Computational studies suggest that arginine binds non-covalently to certain sites on a protein(9). In combination with glutamate, the stabilizing effect of arginine could be further enhanced, probably through a more complex mechanism that involves the formation of arginine-glutamate clusters(10). The small molecule drug dexamethasone phosphate (DMP) was discovered to reduce the formation of bevacizumab aggregates when administered in a co-formulation. Docking studies of DMP on a homology model of bevacizumab suggest that binding to a lysine residue that may be involved in crystal contacts, sterically hinders the formation of protein-protein interfaces and thus inhibits aggregation(11-13). This opposes the finding in another study by hydrogen-deuterium exchange spectroscopy that identifies a patch of residues in the CDR region to be involved in the formation of bevacizumab aggregates at elevated temperatures(14).

\section{$\underline{\text { Virtual Screen }}$}

Here, we present an approach that aims at identifying new compounds that bind to a predicted aggregation hotspot of IFN, thus inhibiting the formation of protein-protein interfaces and subsequently aggregation.

Due to the large, flat interfaces that form during protein-protein interactions, these have long been considered difficult targets for small molecules. More recently however many successful examples have been presented(15). In order to identify small molecules that bind to a defined protein site, a common approach is running a virtual screen, where databases of millions of compounds are tested 
for affinity towards the specified binding site by docking algorithms(16). The database selection is the first step critical to the success of a docking campaign. Not only the database's size is relevant but also its compound diversity and the compounds' availability. The ZINC15 database is one of the largest publicly accessible databases, including more than 700.000 .000 compounds, that can be filtered according to their commercial availability, reactivity or hydrophobicity(17). Glide, Gold or Autodock Vina are some programs to perform high throughput pose prediction and scoring(18-20). While current docking algorithms account for ligand flexibility, the receptor is considered to be rigid, an assumption that can drastically reduce enrichment of active compounds in the highest scoring hits(21). Another limitation of docking comes from not accounting for water thermodynamics appropriately. Further issues when interpreting docking results can arise from an incorrect calculation of protonation states of the binding site. Due to docking's many simplifications and limitations, its results should be considered as a starting point to suggest interesting compounds, rather than a method to elucidate detailed features of protein-ligand interaction, such as binding kinetics and free energies.

\section{Free energy of binding}

A large variety of methods to accurately calculate free energies of binding from atomistic molecular dynamics simulations exist. Unbiased simulations can give detailed information on the binding mechanism, kinetics and secondary binding sites(22). However, they demand large amounts of computational resources. Biased simulations reduce the computational cost by introducing potentials that facilitate the sampling of unfavorable regions in the system's phase space. In the simplest case, a biasing potential can be a harmonic oscillator, restraining the distance between two atoms. In practice, this is employed for example in meta-dynamics or umbrella sampling $(23,24)$. Introducing biasing potentials to a system has been observed to cause dissipation of energy in umbrella sampling simulations(25). This effect has been overcome more recently by accounting for the energy required to attach and release these potentials(26). The resulting attach-pull-release umbrella sampling method has a solid theoretical foundation and has been able to accurately predict free energies of binding in guest-host systems $(27,28)$. 
Experimentally, binding energies can be determined from titration experiments using methods such as isothermal calorimetry, surface plasmon resonance, nuclear magnetic resonance or microscale thermophoresis.

Binding is just one aspect to filter for in a virtual screen. Other physico-chemical properties such as solubility, reactivity and toxicity are equally important to obtain successful candidate compounds.

\section{Additional aspects of virtual screens}

A compounds solubility is typically indicated by its $\log _{10} S$ value, where $S$ is the compounds concentration in the aqueous phase in equilibrium with the most stable form of the crystalline compound(29). They are most commonly predicted by quantitative structure-properties relationship (QSPR) methods, such as group contributions(30,31), neural networks(32) or multiple linear regression analysis(33). A public challenge to predict the solubility of a set of 32 compounds from a training set of 100 molecules revealed the current state of prediction quality: the best performing predictions on a dataset including outliers gave $\mathrm{R}^{2}$ values of approximately 0.6 and approximately $20 \%$ of $\log _{10} S$ values were calculated correctly(34-36). However, solubility predicting methods typically do not consider solution $\mathrm{pH}$ but are only trained against physiological conditions. In formulation science, where $\mathrm{pH}$ and ionic strength can differ strongly from this condition, pKas should therefore also be considered when assessing solubility. A carboxylic acid will for example show different solubilities depending on its protonation state.

A property closely linked to the water solubility is the octanol-water partition coefficient as a measure of hydrophobicity for small molecules(37). The ZINC15 database can conveniently be filtered by predicted $\log _{10} P$ values $(38,39)$.

\section{Experimental assessment of protein stability}

Once a compound passed all filters of the virtual screen, we want to test its effect on protein aggregation experimentally. 
Aggregation processes are typically very slow. To predict the stability of a formulation in a reasonable time frame, one can therefore test a formulation for surrogate endpoints such as e.g. the interaction parameter $k_{d}$ as a measure of colloidal stability or the inflection point (IP) of an unfolding experiment as a measure of conformational stability. Alternatively, stress-studies can be performed, where the formulation is exposed to an aggregation trigger such as freezing/thawing, heat, shaking, shear or light. Light and thermal stress are known to induce chemical changes in the protein that are not the scope of this work $(40,41)$. We therefore apply, heat, freeze-thaw and shaking stress to evaluate the effect of the candidate excipients. To benchmark our compounds, we compare them against L-arginine and $\mathrm{D}(+)$-trehalose, two substances commonly employed as excipients in protein formulation.

\section{Results}

\section{Virtual Screen}

We identified a potential aggregation hotspot at residues L26 and F27 of IFN using Aggrescan3D(8) (Figure 1). The hotspot's score remained unchanged among all 25 available structures, showing little effect of protein dynamics on the calculated propensity. The highest-ranking residue patch was defined as binding site for a subsequent virtual screen. Candidate compounds would ideally bind in proximity to the hotspot, blocking it from driving the formation of a protein-protein interface.

Applying a second solubility filter orthogonal to the ZINC database's internal $\log _{10} P$ filter reduced the number of candidates from 52,980 by $40 \%$ to 33,101 compounds. The compounds were then docked with Maestro's virtual screen workflow using GlideSP and GlideXP. The best scoring compounds were then rescored using the MM-GBSA solvent model. After docking the compounds at increasing levels of precision and conformational sampling, 167 compounds were predicted to bind in the hotspot's proximity. These were inspected visually and five were purchased based on their price and availability (Table 1, Figure 2).

\section{Binding study}


All five compounds tested for binding readily dissolved in the experimental buffer, owed to the rigorous filters applied in the prior virtual screen. Out of the tested compounds, only compound $\mathbf{A}$ and L-arginine were detected to bind to the target (Table 1).

A control run using the fluorescent dye from the protein labeling kit as target showed no dose response. For $\mathrm{A}$, a dissociation constant of $108 \mu \mathrm{M} \pm 24 \mu \mathrm{M}$ was determined, which corresponds to a free energy of binding of $-5.44 \pm 0.13 \mathrm{kcal} / \mathrm{mol}$. The free energy of binding calculated by the APR-US method however was found to be below the measured energy (Figure 3).

\section{Protein self-interaction}

The apparent molecular weight $\left(\mathrm{M}_{\mathrm{w}}\right)$ of IFN was measured in the absence and presence of compound A using static light scattering (SLS). As expected from the choice of $\mathrm{pH}$ and ionic strange, IFN forms aggregates in solution. While the aggregation is concentration dependent for low IFN concentrations, a plateau is reached at approximately $6 \mathrm{mg} / \mathrm{ml}$. Even though the presence of compound $\mathbf{A}$ leads to significant reductions in $\mathrm{M}_{\mathrm{w}}$ (Figure S-1) it does not quantitively break up aggregates.

\section{Forced degradation studies}

In order to assess how the compounds, influence the formation of particles, aggregation of IFN was induced in forced degradation experiments. Sub-visible particles and soluble aggregates were quantified after three freeze-thaw cycles with the 5 formulations containing five different excipients obtained from the virtual screen. Additionally, a negative control was run containing only protein and buffer, but no other stabilizing agent. The only compound to significantly reduce both the formation of soluble aggregates and sub-visible particles was found to be compound $\mathbf{A}$. While compounds $\mathbf{B}$ and C would slightly reduce soluble aggregate formation, they showed no benefit on sub-visible particle count compared to the excipient free control (Figure 4). 
In the next step, IFN was exposed to horizontal shaking stress at different concentrations of compound A. The ligand's concentration range was chosen according to the previously determined dissociation constant. The formation of sub-visible particles shows a strong dose response. At high ligand concentrations, where all protein molecules are bound to $\mathbf{A}$, sub-visible particle formation is at a minimum. With decreasing ligand concentration, the share of unbound protein increases and an increase in sub-visible particles is observed (Figure 4).

As a benchmark test, compound $\mathbf{A}$ was compared to the standard excipients L-arginine and $\mathrm{D}(+)$ trehalose at a concentration of $6.25 \mu \mathrm{M}$. All three compounds readily reduce the formation of subvisible particles. However, compound $\mathbf{A}$ shows a lower particle count than the standard excipients (Figure 4).

In order to rule out that the positive effect of compound A on the protein's stability is due to a nonspecific effect, the surface activity (Table 2) of the compound was measured. While compound $\mathbf{A}$ leads to slightly higher surface pressures than the non-surfactant references, its surface activity is far below that of a typical surfactant polysorbate 20 .

Furthermore, the effect of compound A's L-isomeric form, glycyl-L-asparagine, on particle formation was tested (Figure 4). Compound $\mathbf{A}$ drastically reduces sub-visible particle formation compared to all other tested molecules, surprisingly even slightly lowering particle counts compared to the unstressed sample. Glycyl-L-asparagine does not have a beneficial effect on particle formation compared to the excipient free formulation.

In order to study the target specificity of compound $\mathbf{A}$, its stabilizing effect was tested in combination with a mAb (Figure S-2). Here, all tested compounds reduced particle formation with compound $\mathbf{A}$ performing slightly worse than the benchmark excipients.

While compound $\mathbf{A}$ showed a stabilizing effect on IFN when formulations were exposed to agitation or freezing/thawing, it had no effect on the protein's thermal stability (Table S-1). Neither did any other of the examined compounds. 


\section{Toxicity Prediction}

The VirtualToxLab tool predicts a very low toxicity of compound A. It was predicted not to bind to any of the toxicity related target proteins and its overall toxicity score was found to be 0.079 , ranking for example below vitamin C which has a score of 0.253 .

\section{Discussion}

The virtual screen was successful with a hit rate of $20 \%$ in identifying one out of five tested molecules that bind to IFN with $\mu \mathrm{M}$ affinities. Further improving binding affinities could be achieved by allowing for more hydrophobic compounds in the screen or increasing the compound's size. This may however have a negative effect on toxicity and clearance of the compound. Even though we were successful in identifying a compound that reduces particle formation, docking alone cannot be considered as proof of a structure-activity relationship. While MM-GBSA ranked affinities of compounds $\mathbf{C}$ to $\mathbf{E}$ other than compound $\mathbf{A}$ higher, they were not detected to bind in MST measurements. As already mentioned, this may be explained by the many simplifications made by the docking algorithms.

In order to obtain additional binding molecules, the same library was docked against an ensemble of IFN conformations, leading to the identification of one additional hit, which however showed no increase in stability in any forced degradation study (data not shown). This finding indicates that not all protein-ligand complexes would result in a stabilization, but only specific interactions. When adding the tested compounds to formulations containing mAb-1 instead of IFN, compound A, L-arginine and $\mathrm{D}(+)$-trehalose would all reduce particle formation after freeze-thaw stress to the same extend. Given the structural diversity of the three compounds, stabilization of mAb-1 can be interpreted as a nonspecific effect. The non-specific stabilization observed with a mAb and the non-stabilizing effect of compound A's enantiomer with IFN both strongly support our initial hypothesis of a specific proteinligand interaction leading to a stabilization against native protein aggregation of IFN. It is important to point out that the stabilizing effect of compound $\mathbf{A}$ may very well be $\mathrm{pH}$ dependent, especially due to its multiple titratable sites which could result in a pH dependent protein-ligand interaction profile(42). 
The APR-US calculations show that the affinity towards the stipulated binding site is far below the measured one (Figure 3). This may indicate the presence of additional binding sites with higher affinities towards the ligand. The presence of multiple binding sites could be confirmed by unrestrained simulations adding up to a free energy of binding in the same range as the one measured by MST (to be published). Limitations arise from using fixed protonation states for both the ligand and the protein, even though interactions between conformations, protein-ligand interactions and protonation states are well described. Taking these factors into account e.g. by constant pH MD simulations would however further increase the already large computational cost of these simulations.

A search in the BindingDB database for compounds with binding energies between -3 and $-2 \mathrm{kcal} / \mathrm{mol}$ results in multiple Guest-Host systems, with guests similar in structure and size to compound $\mathbf{A}$. Compounds in the -6 to $-5 \mathrm{kcal} / \mathrm{mol}$ range tend to be more hydrophobic and/or larger(43). This indicates that the actual binding mechanism may be more complex than initially suggested.

Even though we were successful in identifying a stabilizing compound, it is important to point out that we readily relied on assumptions regarding the identification of aggregation prone regions and the binding site that have yet to be proven. A3D does not take the electrostatics surrounding hydrophobic patches into account and was only tested on a limited amount of proteins. The differences in the stabilizing effect of the compounds depending on the nature of the force degradation study indicate a vague definition of the concept of an aggregation prone region and poses a drastic simplification to a complex phenomenon. Heat induced aggregation has been shown to induce non-native aggregation involving partial unfolding of the protein. While the compound $\mathbf{A}$ was shown to bind to IFN, it would not lead to a conformational stabilization as indicated by measurements of IP and $\mathrm{T}_{\text {onset. }}$. The stabilizing effect of compound $\mathbf{A}$ was confirmed in a horizontal shaking stress study, ruling out a change in ice crystal formation as origin of the observation. Measurements of the compounds surface activity do not indicate a high affinity towards interfaces. Together with the observed decrease in apparent $M_{w}$ from the SLS measurements in the presence of compound $\mathbf{A}$, it supports our hypothesis of an inhibition of sub-visible particle formation by impeding the formation of specific native protein-protein contacts. 
Previous studies have already shown the existence of a stress-structure interaction(14). This poses a set-back to our approach, since a novel excipient stabilizing only against a certain type of stress is not ideal. It can therefore only be considered a hypothesis that the selection of the binding site is related to the observed effects. The actual binding mechanism of compound $\mathbf{A}$ has to be determined experimentally. Due the self-association of IFN at pH 7.0, this cannot be achieved by NMR but possibly by crystallographic methods. Given these insights, it seems sensible to favor ligand-based approaches opposed to our receptor-based approach. Establishing relevant stability indicating assays remains however a topic of ongoing research(44).

Given the proximity of the hotspot to the IFN's receptor binding site, binding kinetics and clearance of the excipient are highly relevant for an in-vivo application. A dissociation rate of the ligand that would limit the formation rate of the drug-target complex, i.e. a high residence time of the protein-excipient complex, will alter the drug's efficacy. We are currently looking into in-silico methods for the calculation of $\mathrm{k}_{\mathrm{on}} / \mathrm{k}_{\text {off }}$ and in-vitro activity assays.

For drug products, toxicity of the excipient candidates remains a critical point. A specifically designed database containing only compounds with a proven record of low toxicity could help to overcome this problem. Considering however the low hit rate in the virtual screen, further limiting the screened chemical space might cause the elimination of any potential binders. Additional in-silico methods to predict toxicity can be considered, always taking resulting metabolites into consideration. Nevertheless, the discovered compound could immediately be used in diagnostic devices without the need for additional toxicity studies. While IFN is currently not a typical reagent in diagnostics, our approach can easily be transferred to any other relevant protein.

\section{Conclusion}

Here, we describe a structure-based approach that was successful in discovering a small organic molecule that stabilizes Interferon-alpha-2a and confirmed the hypothesis that the formation of a protein-ligand complex can lead to an inhibition of aggregation and particle formation. Our systematic 
approach helped us to narrow down a database of millions of compounds to merely five. The compound glycyl-D-asparagine reduces the formation of sub-visible particles and soluble aggregates after freeze-thaw and agitation stress in a concentration dependent manner that correlates well with its binding affinity towards IFN. It shows higher stabilizing activity than its enantiomer glycyl-Lasparagine and the standard excipients L-arginine and $\mathrm{D}(+)$-trehalose. We gave a new use to tools that are developed with small molecule drug discovery in mind and show how they can be applied to therapeutic protein formulation development. While a drug discovery campaign targets protein linked to a disease, here the drug protein itself is the target.

\section{Methods}

\section{$\underline{\text { Virtual Screen }}$}

A homology model of IFN was generated based on the PDB entry 4Z5R using Modeller(45). A potential aggregation hotspot was identified by submitting the homology model to the Aggrescan3D server(8).

The protein structure of IFN was prepared for docking using Maestro's (Schrödinger, Inc., New York, New York, USA) protein preparation wizard with $\mathrm{pH}$ set to 7.0. Maestro was used to generate a docking grid using the residues that are located in the identified aggregation hotspot as grid center. The ZINC15 database tranches were selected to include only compounds with a $\log _{10} P \leq-1$, "in-stock" availability and standard reactivity. The compounds were then prepared for docking using LigPrep as implemented in Maestro. Qikprop was used to predict the compounds physicochemical properties and only compounds with a $\log _{10} S$ value $\geq-1$ were retained. All compounds were then docked with Glide HT. The best scoring $10 \%$ were then redocked and scored with GlideSP. The best scoring $10 \%$ were redocked and rescored using GlideXP and up to 3 poses per compound were generated. These poses were rescored using the Prime MM-GBSA model. We then looked manually for substances available for purchase below $200 € / g$.

\section{Sample Preparation}


An aqueous bulk solution of Interferon-alpha-2a (Roche, Penzberg) was dialysed (Spectra-Por) into $50 \mathrm{mM} \mathrm{Pi}$ (di-Sodium hydrogen phosphate dihydrate: VWR Chemicals, Leuven, Sodium dihydrogenphosphate dihydrate: Grüssing $\mathrm{GmbH}$, Filsum) buffer at $\mathrm{pH}$ 7.0. The solution was filtered using a $0.22 \mu \mathrm{m}$ cellulose acetate filter (VWR Chemicals, Leuven), which were shown to be low protein binding(46). A protein concentration of $1.4 \mathrm{mg} / \mathrm{ml}$ was obtained as determined by measuring the light absorption at $280 \mathrm{~nm}$ using a NanoDrop (Thermo Fisher Scientific, Waltham, MA, USA).

Excipient stock solutions were prepared by dissolving the excipient in $50 \mathrm{mM} \mathrm{Pi}$ buffer at pH 7.0 and adjusting the $\mathrm{pH}$ to 7.0 as required either with hydrochloric acid or concentrated sodium hydroxide. Buffer was then added to obtain a final excipient concentration of $500 \mathrm{mM}$. The excipient stock solution was then filtered using a $0.22 \mu \mathrm{m}$ filter (VWR Chemicals, Leuven).

\section{Binding study}

Binding affinities were determined by microscale thermophoresis (Monolith, NanoTemper, Munich, Germany). Interferon-alpha-2a was labelled fluorescently (Monolith Protein Labeling Kit RED-NHS) and excipient candidates were titrated using $50 \mathrm{mM}$ phosphate buffer at $\mathrm{pH} 7.0$ with a polysorbate 20 (Sigma Aldrich) concentration of $0.05 \%(47)$. Excitation-power was set to $20 \%$ and MST-power was set to "high".

\section{Molecular dynamics simulations}

The best scoring pose of the MM-GBSA rescoring served as input structure to calculate free energies of binding by the Attach-Pull-Release (APR) Umbrella Sampling approach(26-28). The PDB structure generated by the virtual screen, containing the ligand docked to the protein, was reoriented using the z-align script from the APR suite. Restraints were gradually attached in 13 windows and the distance between the compound and its binding site was gradually increased in 46 windows. For the first window of the attachment phase where the APR restraints are set to 0 , an additional distant restraint was implemented to define the binding site and avoid the ligand leaving. The systems for each window were constructed using tleap, adding 20500 water molecules to each system, using the APR procedure. 
The CUDA implementation of pmemd in Amber16 was used along with the ff14SB, GAFF2 and TIP3P force-fields $(48,49)$. The ligand was parametrized using GAFF2 for bonded and non-bonded parameters. Atomic partial charges were calculated with Gaussian 16 (Gaussian Inc., Wallingford, CT, U.S.A.) and fitted with the RESP procedure in antechamber. Hydrogen mass repartitioning and the SHAKE algorithm were used to allow timesteps of $4 \mathrm{fs}(50,51)$. Pressure was regulated using a Monte Carlo barostat and a Langevin thermostat was used to keep the temperature at 298.15 K. Modifications to the APR script were implemented to allow parallel runs of the respective windows on the GPU cluster and facilitate system preparation. The simulation time in each window was $112.5 \mathrm{~ns}$ resulting in approximately $6.6 \mu$ s total simulation time. Calculation of the free energy of binding was performed by using the thermodynamic integration scheme as implemented in the APR script.

\section{$\underline{\text { Toxicity Prediction }}$}

The toxicity for the candidate compound A was predicted using OpenVirtualToxLab(52).

\section{Forced degradation studies}

Each replicate sample was filled in a separate 2 R vial (Fiolax, klar HGA 1/ISO 720). The vials were capped and crimped pneumatically. Excipients and buffer were spiked into the IFN solution to obtain a final formulation of $1 \mathrm{mg} / \mathrm{ml}$ of protein, $50 \mathrm{mM}$ excipient, $50 \mathrm{mM}$ Pi at $\mathrm{pH} 7.0$.

To evaluate the stabilizing impact of the excipient candidates, samples were frozen and thawed three times in a Christ 2D-6 freeze dryer. A temperature ramp of $1 \mathrm{~K} / \mathrm{min}$ and a hold time of $2 \mathrm{~h}$ were used. The protein was also exposed to shaking stress using a horizontal shaker (IKA HS 260 basic, 300 rpm). Sub-visible particles were detected by flow imaging (FlowCam, Fluid Imaging Technologies, Inc., Scarborough, ME, USA). Soluble aggregates were detected by size-exclusion chromatography on a Dionex Summit HPLC system at $280 \mathrm{~nm}$ using a Superose 12 10/300 GL as stationary phase (GE Healthcare Life Sciences, Chalfont St Giles, UK) and $50 \mathrm{mM} \mathrm{Pi}, 200 \mathrm{mM} \mathrm{NaCl}, \mathrm{pH} 7.0$ as mobile phase. 
Heat induced degradation was measured with by nanoDSF and backscattering (Prometheus NT.48, NanoTemper, Munich, Germany) at a heating rate of $1{ }^{\circ} \mathrm{C} / \mathrm{min}$ from 25 to $95^{\circ} \mathrm{C}$ in standard capillaries (NanoTemper, Munich, Germany).

\section{$\underline{\text { Apparent } M_{w}}$}

Apparent $M_{w}$ was measured by static light scattering (DynaPro III, Wyatt Technology Europe, Dernbach, Germany) in a 1536 well plate (Aurora Microplates, Whitefish, MT, USA) with $8 \mu$ l of sample volume and $3 \mu \mathrm{l}$ of silicon oil (Alfa Aesar, ThermoFisher $\mathrm{GmbH}$, Kandel, Germany). The well plate was calibrated with a dilution series of dextran (Sigma-Aldrich Chemie GmbH, Taufkirchen, Germany). Due to the sensitivity of light scattering to larger particles, stock solutions were additionally filtered using $0.02 \mu \mathrm{m}$ filters (Whatman, GE Healthcare UK, Buckinghamshire, UK)

\section{Acknowledgements}

This work is part of a project that has received funding from the European Union's Horizon 2020 research and innovation program under the Marie Skłodowska-Curie grant agreement No. 675074. The authors are grateful to NanoTemper for kindly providing measurement time and consumables. Molecular dynamics simulations were carried out on the Steno GPU cluster at DTU chemistry. We want to acknowledge Jonas Mansoor's contribution to setting up and administrating the GPU cluster. Quantum mechanical calculations were carried out on the Linux Cluster of the Leibniz Rechenzentrum. We are grateful to Dr. Thomas Wein for fruitful discussions and to Luis Sánchez for his help with surface pressure measurements.

\section{Competing interests}

The authors declare no competing interests.

\section{Corresponding authors}

Correspondence to Andreas Tosstorff. 


\section{References}

1. Ratanji KD, Derrick JP, Dearman RJ, Kimber I (2014) Immunogenicity of therapeutic proteins: Influence of aggregation. J Immunotoxicol 11(2):99-109.

2. Wang W (2015) Advanced protein formulations. Protein Sci 24(7):1031-9.

3. Roberts CJ (2014) Protein aggregation and its impact on product quality. Curr Opin Biotechnol 30(0):211-217.

4. Clackson T, Wells J a (1995) A hot spot of binding energy in a hormone-receptor interface. Science (80- ) 267(5196):383-386.

5. Fernandez-Escamilla A-M, Rousseau F, Schymkowitz J, Serrano L (2004) Prediction of sequence-dependent and mutational effects on the aggregation of peptides and proteins. Nat Biotechnol 22(10):1302-1306.

6. Conchillo-Solé O, et al. (2007) AGGRESCAN: a server for the prediction and evaluation of "hot spots" of aggregation in polypeptides. BMC Bioinformatics 65(8). doi:10.1186/1471-2105-865.

7. Trovato A, Seno F, Tosatto SCE (2007) The PASTA server for protein aggregation prediction. Protein Eng Des Sel 20(10):521-523.

8. Zambrano R, et al. (2015) AGGRESCAN3D (A3D): Server for prediction of aggregation properties of protein structures. Nucleic Acids Res 43(W1):W306-W313.

9. Barata TS, Zhang C, Dalby PA, Brocchini S, Zloh M (2016) Identification of protein-excipient interaction hotspots using computational approaches. Int J Mol Sci 17(6). doi:10.3390/ijms17060853.

10. Kheddo P, et al. (2014) The effect of arginine glutamate on the stability of monoclonal antibodies in solution. Int J Pharm 473:126-133. 
11. Veurink M, Westermaier Y, Gurny R, Scapozza L (2013) Breaking the aggregation of the monoclonal antibody bevacizumab (Avastin ${ }^{\circledR}$ ) by dexamethasone phosphate: Insights from molecular modelling and asymmetrical flow field-flow fractionation. Pharm Res 30(4):11761187.

12. Westermaier $\mathrm{Y}$, et al. (2013) Identification of aggregation breakers for bevacizumab (Avastin ${ }^{\circledR}$ ) self-association through similarity searching and interaction studies. Eur J Pharm Biopharm 85(3 PART A):773-780.

13. Veurink M, Stella C, Tabatabay C, Pournaras CJ, Gurny R (2011) Association of ranibizumab (Lucentis ${ }^{\circledR}$ ) or bevacizumab (Avastin ${ }^{\circledast}$ ) with dexamethasone and triamcinolone acetonide: An in vitro stability assessment. Eur J Pharm Biopharm 78(2):271-277.

14. Zhang A, Singh SK, Shirts MR, Kumar S, Fernandez EJ (2012) Distinct aggregation mechanisms of monoclonal antibody under thermal and freeze-thaw stresses revealed by hydrogen exchange. Pharm Res 29(1):236-250.

15. Jin L, Wang W, Fang G (2014) Targeting Protein-Protein Interaction by Small Molecules. Annu Rev Pharmacol Toxicol 54(1):435-456.

16. Shoichet BK (2004) Virtual screening of chemical libraries. Nature 432(7019):862-865.

17. Sterling T, Irwin JJ (2015) ZINC 15 - Ligand Discovery for Everyone. J Chem Inf Model 55(11):2324-2337.

18. Repasky MP, et al. (2012) Docking performance of the glide program as evaluated on the Astex and DUD datasets: A complete set of glide SP results and selected results for a new scoring function integrating WaterMap and glide. J Comput Aided Mol Des 26(6):787-799.

19. Trott O, Olson A (2010) Autodock vina: improving the speed and accuracy of docking. J Comput Chem 31(2):455-461.

20. Jones G, Willett P, Glen RC, Leach AR, Taylor R (1997) Development and validation of a genetic 
algorithm for flexible docking. J Mol Biol 267(3):727-748.

21. Korb O, et al. (2012) Potential and limitations of ensemble docking. J Chem Inf Model 52(5):1262-1274.

22. Buch I, Giorgino T, De Fabritiis G (2011) Complete reconstruction of an enzyme-inhibitor binding process by molecular dynamics simulations. Proc Natl Acad Sci U S A 108(25):101849.

23. Barducci A, Bonomi M, Parrinello M (2011) Metadynamics. Wiley Interdiscip Rev Comput Mol Sci 1(5):826-843.

24. Woo H-J, Roux B (2005) Calculation of absolute protein-ligand binding free energy from computer simulations. Proc Natl Acad Sci U S A 102(19):6825-30.

25. Velez-Vega C, Gilson MK (2012) Force and stress along simulated dissociation pathways of cucurbituril-guest systems. J Chem Theory Comput 8(3):966-976.

26. Velez-Vega C, Gilson MK (2013) Overcoming dissipation in the calculation of standard binding free energies by ligand extraction. J Comput Chem 34(27):2360-2371.

27. Gilson MK, Given JA, Bush BL, McCammon JA (1997) The statistical-thermodynamic basis for computation of binding affinities: A critical review. Biophys J 72:1047-1069.

28. Henriksen NM, Fenley AT, Gilson MK (2015) Computational Calorimetry: High-Precision Calculation of Host-Guest Binding Thermodynamics. J Chem Theory Comput 11(9):4377-4394.

29. Jorgensen WL, Duffy EM (2002) Prediction of drug solubility from structure. Adv Drug Deliv $\operatorname{Rev} 54: 355-366$.

30. Klopman G, Wang S, Balthasar DM (1992) Estimation of Aqueous Solubility of Organic Molecules by the Group Contribution Approach. Application to the Study of Biodegradation. J Chem Inf Comput Sci 32:474-482. 
31. Kühne R, Ebert R-U, Kleint F, Schmidt G, Schüürmann G (1995) Group contribution methods to estimate water solubility of organic chemicals. Chemosphere 30(11):2061-2077.

32. Tetko I V, Tanchuk VY, Kasheva TN, Villa AEP (2001) Estimation of Aqueous Solubility of Chemical Compounds Using E-State Indices. J Chem Inf Comput Sci 41(6):1488-1493.

33. Duffy EM, Jorgensen WL (2000) Prediction of Properties from Simulations: Free Energies of Solvation in Hexadecane, Octanol, and Water. J Am Chem Soc 122(12):2878-2888.

34. Hewitt M, et al. (2009) In silico prediction of aqueous solubility: The solubility challenge. J Chem Inf Model 49(11):2572-2587.

35. Llinàs A, Glen RC, Goodman JM (2008) Solubility challenge: Can you predict solubilities of 32 molecules using a database of 100 reliable measurements? J Chem Inf Model 48(7):12891303.

36. Hopfinger AJ, Esposito EX, Llinàs A, Glen RC, Goodman and JM (2009) Findings of the Challenge To Predict Aqueous Solubility Anton. J Chem Inf Model 49:1-5.

37. Lipinski CA, Lombardo F, Dominy BW, Feeney PJ (2001) Experimental and computational approaches to estimate solubility and permeability in drug discovery and development. $A d v$ Drug Deliv Rev 46:3-26.

38. Sterling T, Irwin JJ (2015) ZINC 15 - Ligand Discovery for Everyone. J Chem Inf Model 55(11):2324-2337.

39. Irwin JJ, Shoichet BK (2005) ZINC - A Free Database of Commercially Available Compounds for Virtual Screening. J Chem Inf Model 45(1):177-182.

40. Vanhooren A, Devreese B, Vanhee K, Van Beeumen J, Hanssens I (2002) Photoexcitation of tryptophan groups induces reduction of two disulfide bonds in goat $\alpha$-lactalbumin. Biochemistry 41(36):11035-11043. 
41. Brange J, Havelund S, Hougaard P (1992) Chemical Stability of Insulin. 2. Formation of Higher Molecular Weight Transformation Products During Storage of Pharmaceutical Preparations. Pharm Res 9(6):727-734.

42. Nuhu MM, Curtis $\mathrm{R}$ (2015) Arginine dipeptides affect insulin aggregation in a pH- and ionic strength-dependent manner. Biotechnol J 10(3):404-416.

43. Gilson MK, et al. (2016) BindingDB in 2015: A public database for medicinal chemistry, computational chemistry and systems pharmacology. Nucleic Acids Res 44(D1):D1045-D1053.

44. Svilenov H, Markoja U, Winter G (2018) Isothermal chemical denaturation as a complementary tool to overcome limitations of thermal differential scanning fluorimetry in predicting physical stability of protein formulations. Eur J Pharm Biopharm 125:106-113.

45. Andrej Šali (1993) MODELLER A Program for Protein Structure Modeling. Comp protein Model by Satisf Spat restraints:779-815.

46. Mahler H-C, et al. (2010) Adsorption Behavior of a Surfactant and a Monoclonal Antibody to Sterilizing-Grade Filters. J Pharm Sci 99(6):2620-2627.

47. Jerabek-Willemsen M, Wienken CJ, Braun D, Baaske P, Duhr S (2011) Molecular Interaction Studies Using Microscale Thermophoresis. Assay Drug Dev Technol 9(4):342-353.

48. Salmon-Ferrer R, Goetz AW, Poole D, Le Grand S, Walker RC (2013) Routine microsecond molecular dynamics simulations with AMBER - Part II: Particle Mesh Ewald. J Chem Theory Comput 9(9):3878-3888.

49. Jorgensen WL, Chandrasekhar J, Madura JD, Impey RW, Klein ML (1983) Comparison of simple potential functions for simulating liquid water William. J Chem Phys 79(2):926-935.

50. Ryckaert J-P, Ciccotti G, Berendsen HJ. (1977) Numerical integration of the cartesian equations of motion of a system with constraints: molecular dynamics of n-alkanes. J Comput Phys 23(3):327-341. 
51. Feenstra KA, Hess B, Berendsen HJC (1999) Improving efficiency of large time-scale molecular dynamics simulations of hydrogen-rich systems. J Comput Chem 20(8):786-798.

52. Vedani A, Dobler M, Hu Z, Smieško M (2015) OpenVirtualToxLab-A platform for generating and exchanging in silico toxicity data. Toxicol Lett 232(2):519-532. 
Tables

\section{Abbreviations}

\begin{tabular}{|l|l|}
\hline A3D & Aggrescan3D \\
\hline APR & Attach-Pull-Release \\
\hline CUDA & Compute Unified Device Architecture \\
\hline DMP & Dexamethasone phosphate \\
\hline ff14SB & Amber protein force field \\
\hline GAFF2 & General Amber force field 2 \\
\hline GIST & Grid inhomogeneous solvation theory \\
\hline GPU & Graphical processing unit \\
\hline IFN & Interferon-alpha-2a \\
\hline IP & Infliction point of temperature dependent fluorescence signal curve \\
\hline MD & Molecular dynamics \\
\hline MM-GBSA & Molecular mechanics - generalized born surface area \\
\hline MST & Protein database \\
\hline M ${ }_{w}$ & Microscale Thermophoresis \\
\hline PDB & Molecular weight \\
\hline RESP & Temperatic light scattering \\
\hline
\end{tabular}


Table 1: List of purchased compounds

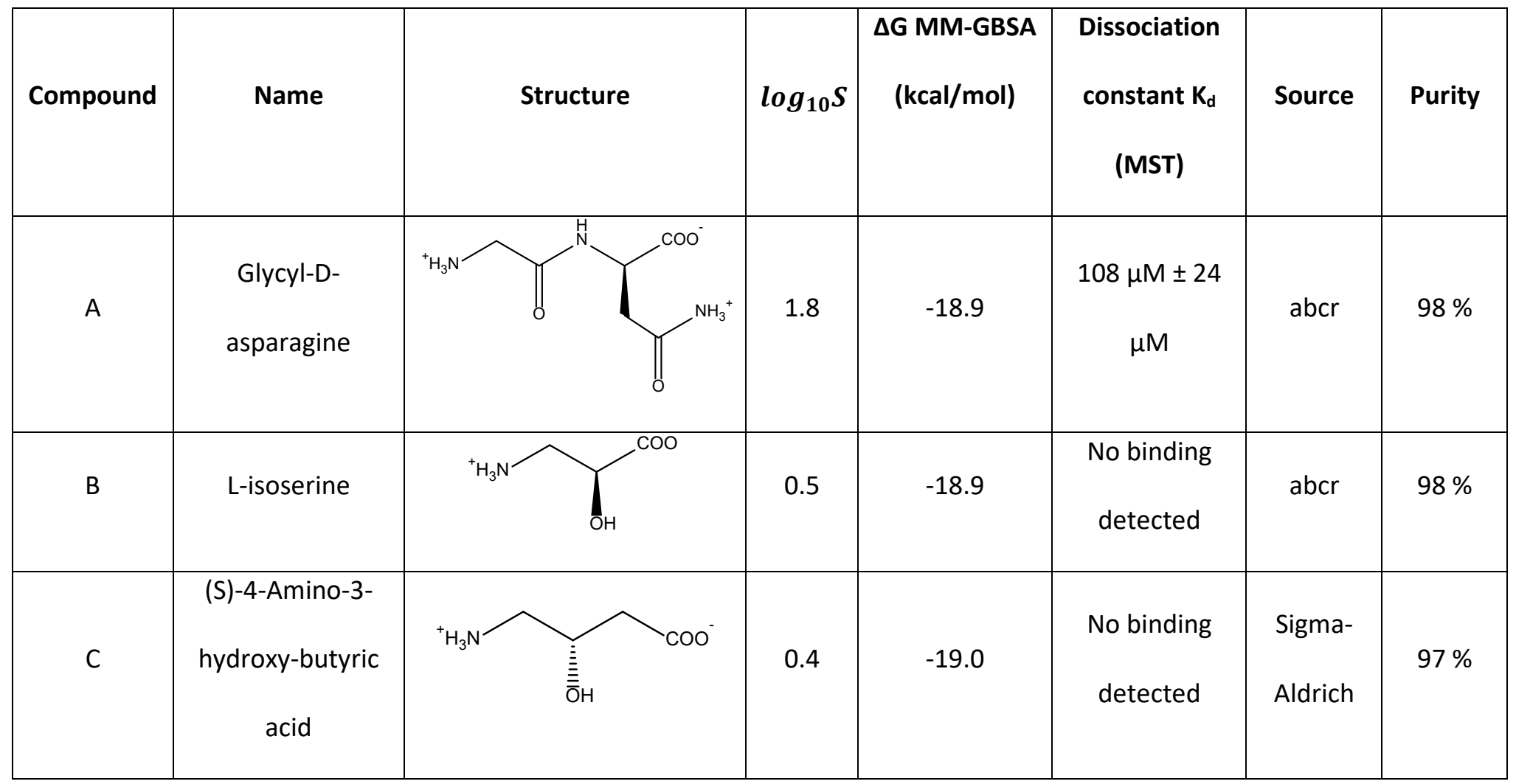




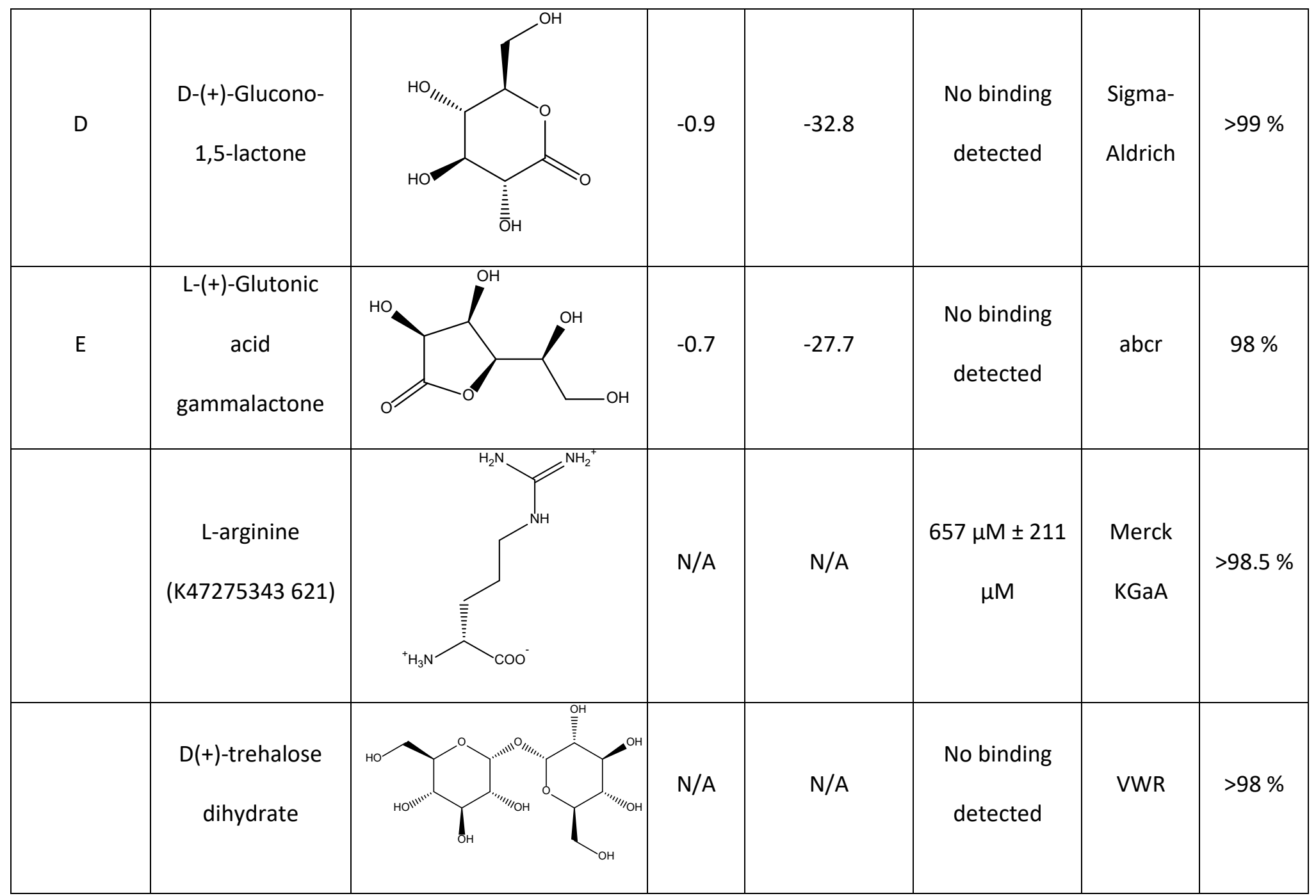


Table 2: Surface pressure data for different excipients. Excipient concentration was 50 mM, except for Tween 20, for which it was $0.005 \% \mathrm{v} / \mathrm{v}$. All measurements were done twice. The errors given correspond to the standard deviations.

\begin{tabular}{|c|c|}
\hline Excipient & Surface pressure $(\mathrm{mN} / \mathrm{m})$ \\
\hline Buffer & $1.7 \pm 0.2$ \\
\hline NaCl & $1.7 \pm 0$ \\
\hline L-arginine & $3.25 \pm 0.15$ \\
\hline D(+)-trehalose & $2.1 \pm 1.6$ \\
\hline Glycerol & $4.75 \pm 0.95$ \\
\hline Polysorbate 20 [0.005\%] & $34.7 \pm 1$ \\
\hline Compound A & $9.0 \pm 0.5$ \\
\hline
\end{tabular}

\section{Figures}

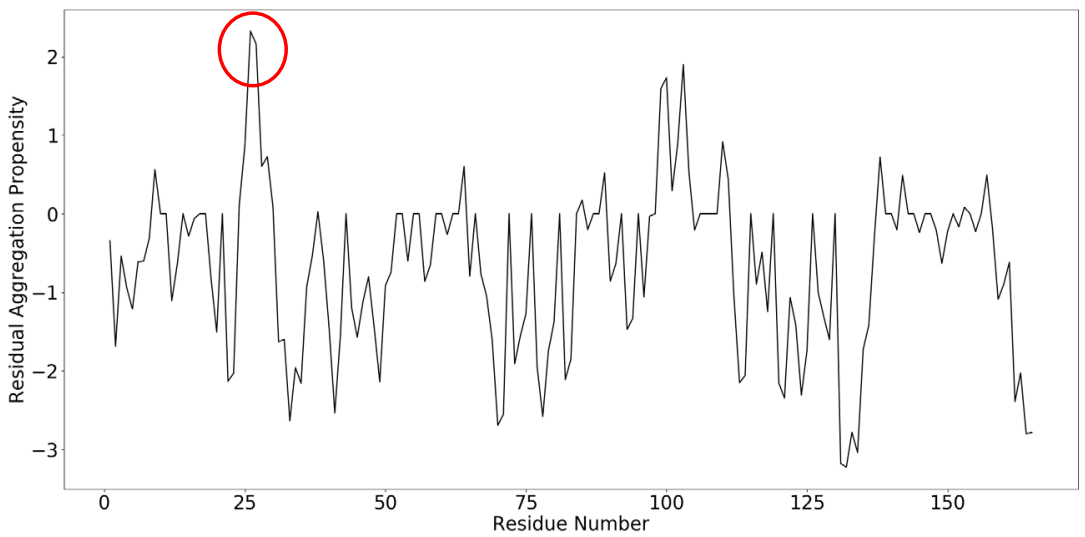

b

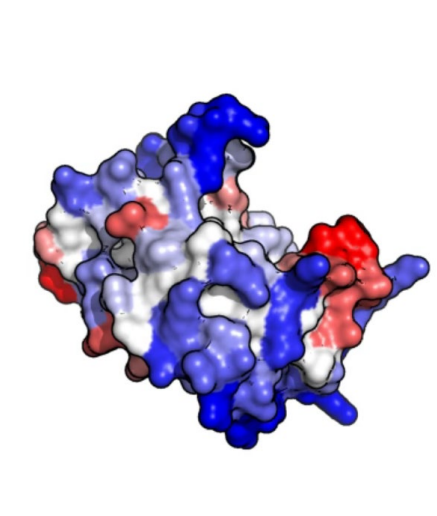

Figure 1: a: Residual aggregation propensity determined using Aggrescan3D. Highest scoring hotspot highlighted with a red circle. b: Visualization of residual aggregation propensity (Blue: low propensity, Red: high propensity). 


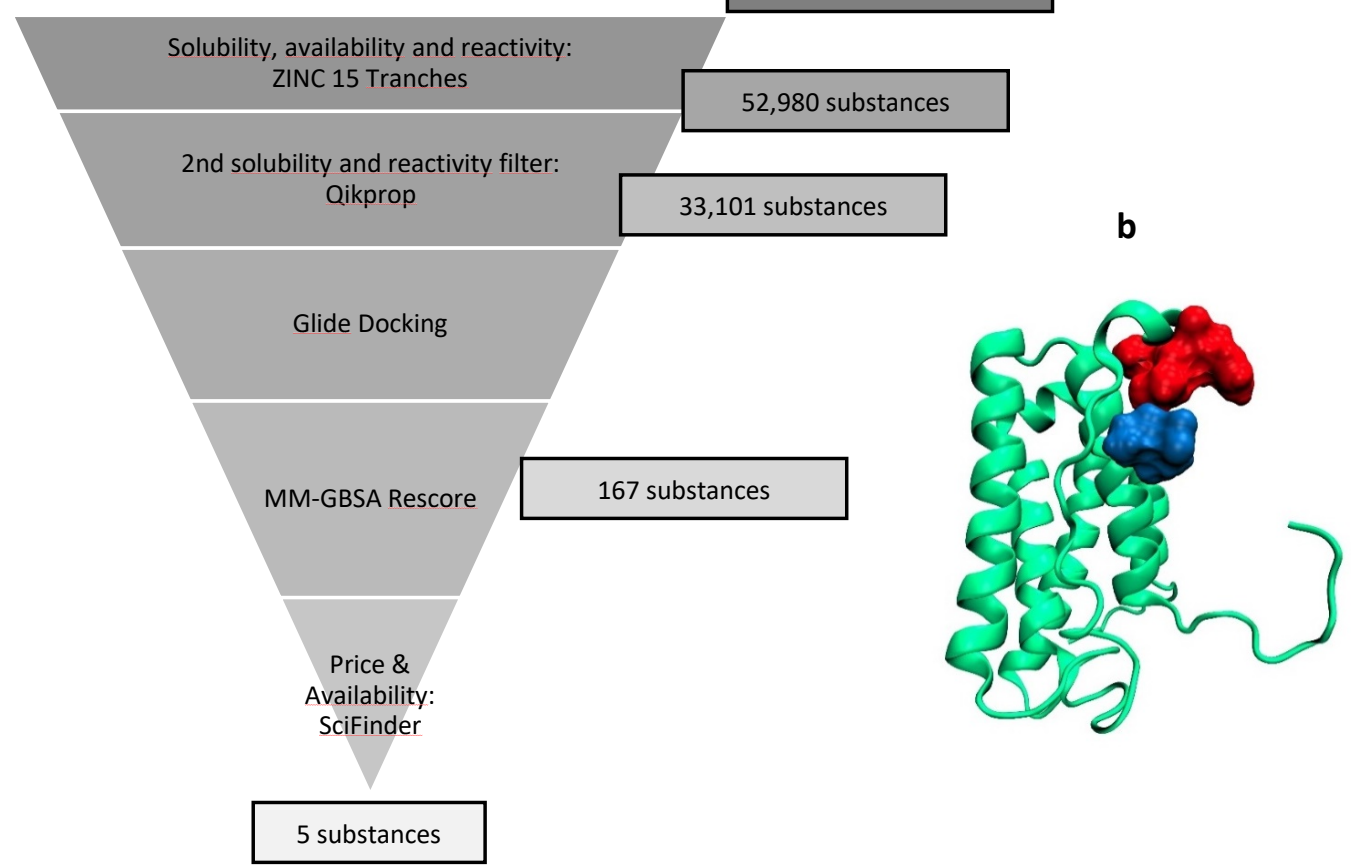

Figure 2: Virtual Screen. Left: Scheme of the virtual screen, designed to identify substances that possess high solubility, low reactivity and high affinity towards the defined binding site. Right: visualization of a ligand (blue) bound to IFN (green) in proximity to the aggregation hotspot predicted by Aggrescan3D (red). 
a

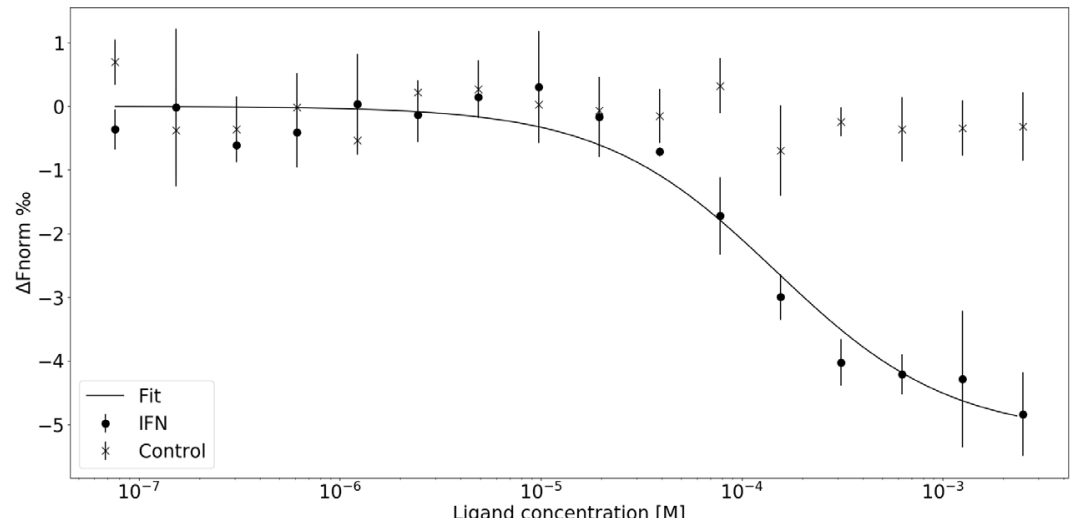

b

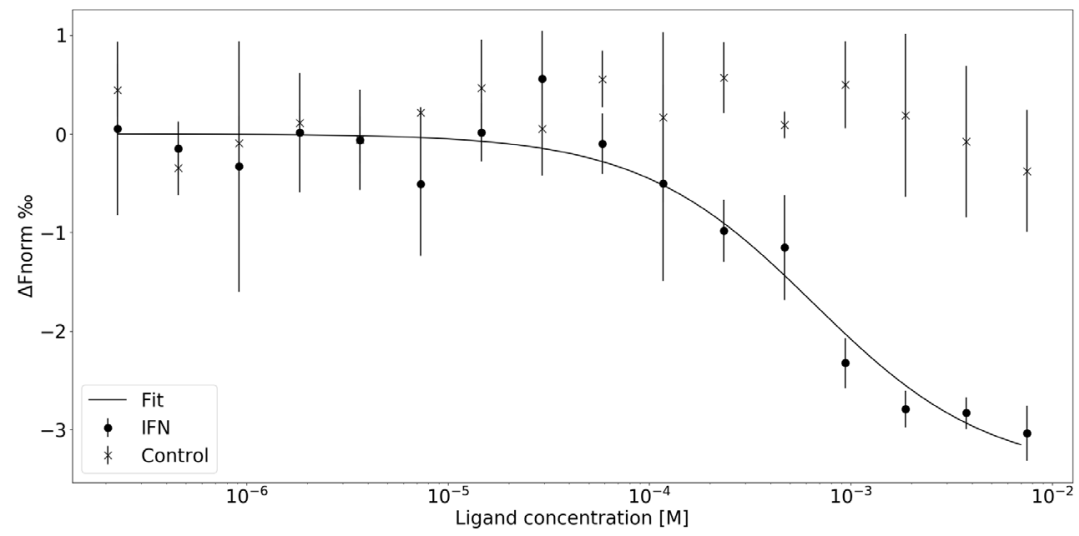

C

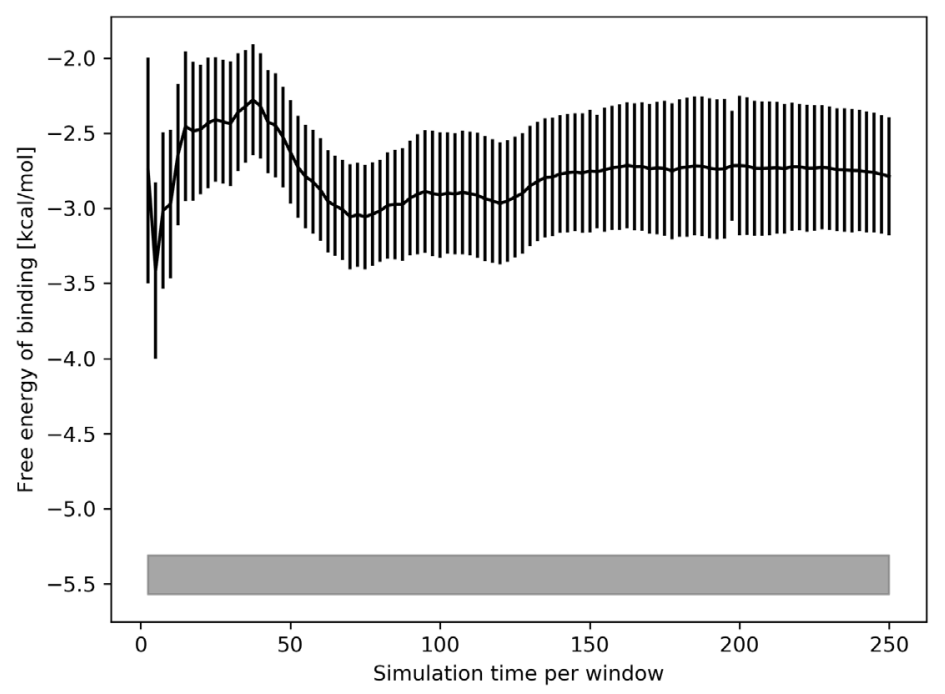

Figure 3: Experimental and calculated binding affinities. a: Dose response curve of $\boldsymbol{A}$ targeting IFN (dots) and the control dye (crosses) as determined by MST: $K d=108 \mu \mathrm{M} \pm 24 \mu \mathrm{M} .50 \mathrm{mM} \mathrm{Pi,} \mathrm{pH} \mathrm{7.0,0.05 \%} \mathrm{Tween} \mathrm{20,} \mathrm{N=3,} \mathrm{IR} \mathrm{intensity=high.}$ Error bars represent the standard deviation of the measurement of three independent samples. $\boldsymbol{b}$ : Dose response curve of L-arginine targeting IFN (dots) and the control dye (crosses) as determined by MST: $K d=657 \mu M \pm 211 \mu M .50 \mathrm{mM} P i$, $p H$ 7.0, 0.05\% Tween 20, N=3, IR intensity=high. Error bars represent the standard deviations of the measurement of three independent samples. c: Black curve: Free energy of binding as calculated by the APR-US method. Error bars 
represent the standard error of the mean. Grey bar: Free energy of binding as determined by MST. The bar's thickness indicates the $68 \%$ confidence range. 
a

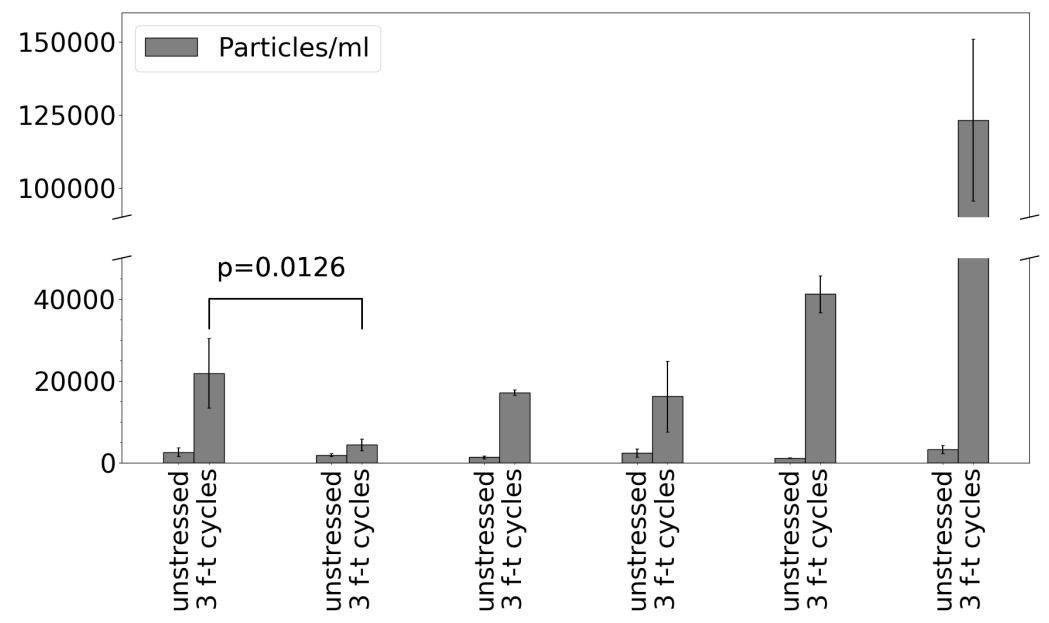

b

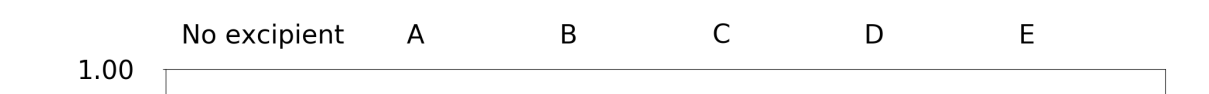

C

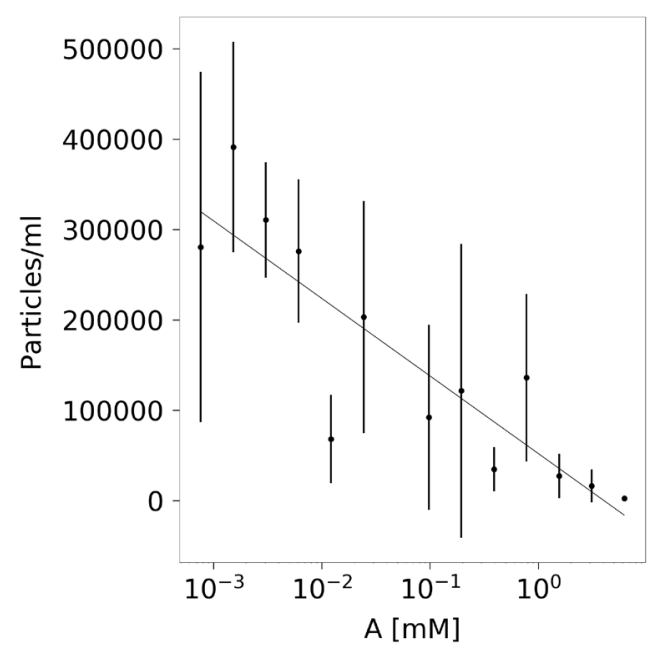

d

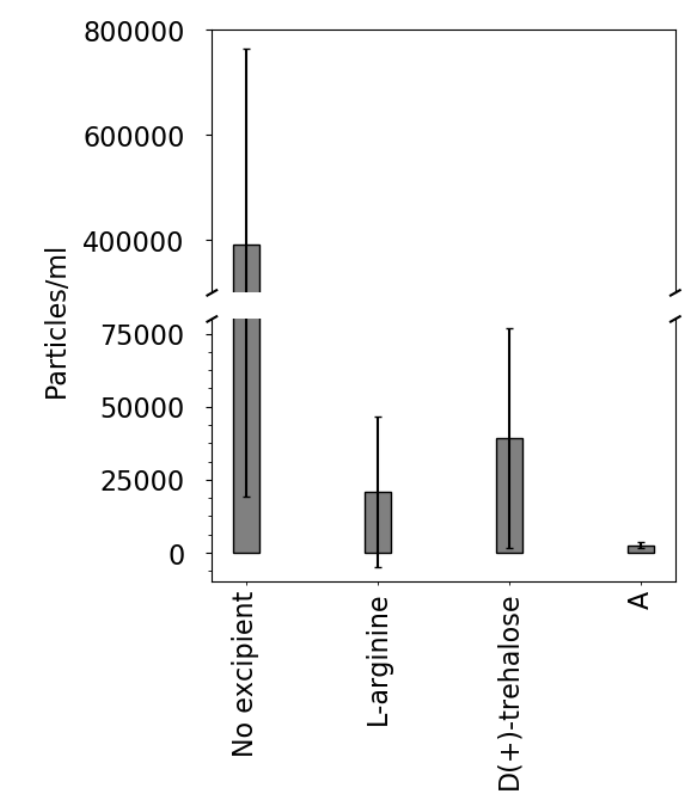


e

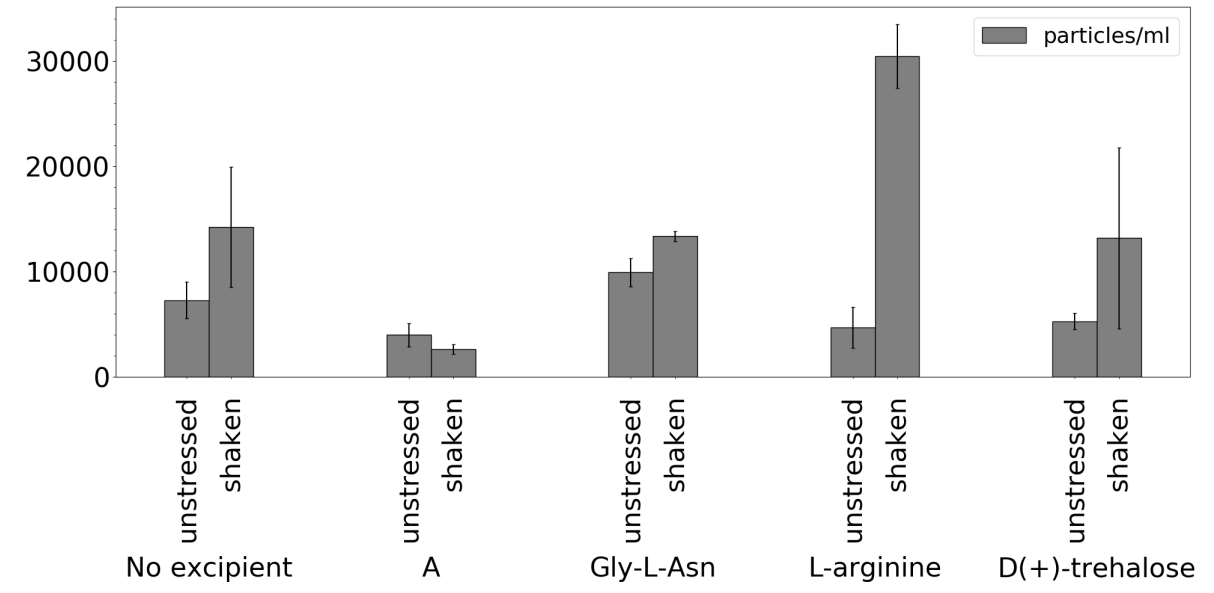

Figure 4: Forced degradation studies. a: Count of particles $\geq 1 \mu \mathrm{m}$ after three cycles of freezing and thawing of IFN formulations. b: Soluble high molecular weight species after three cycles of freezing and thawing of IFN formulations. A-E corresponds to the compounds from Table 1. c: Dependence of sub-visible particle count on A concentration after horizontal shaking. The line is a guide for the eye. $\boldsymbol{d}$ : Sub-visible particle count for $\boldsymbol{A}$ and standard excipients at $6.25 \mathrm{mM}$ after horizontal shaking. e: Sub-visible particle count after submitting a formulation of IFN to $60 \mathrm{~h}$ of horizontal agitation stress. Error bars represent the standard deviations of the measurements of three independent samples. 


\section{Supporting Information}

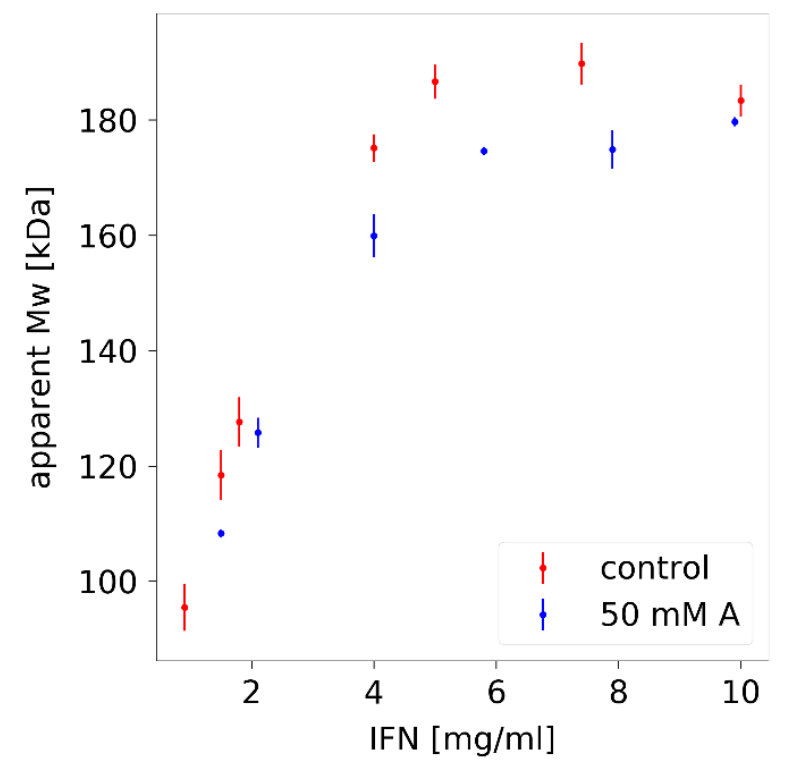

S-Figure 1: Apparent Mw. Measured for different IFN concentrations in presence and absence of $\boldsymbol{A}$ as determined by SLS. Error bars represent the standard deviations of the measurements of three independent samples.

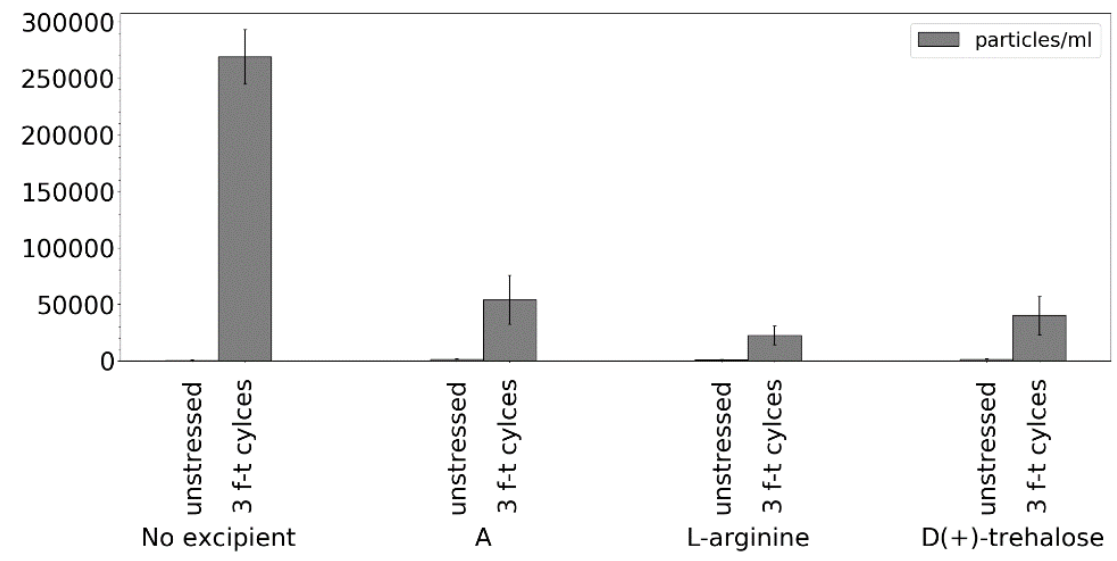

S-Figure 2: Sub-visible particle count before and after submitting a formulation of mAb-1 to three freeze-thaw cycles. 
Table S-1: Inflection point (IP) and aggregation onset temperatures $T_{\text {onset }}$ of IFN formulations. $1 \mathrm{mg} / \mathrm{ml}$ IFN, $50 \mathrm{mM}$ excipient,

$50 \mathrm{mM}$ Pi, pH 7.0.

\begin{tabular}{|c|c|c|}
\hline Excipient & IP $\left[{ }^{\circ} \mathbf{C}\right]$ & Tonset $\left[{ }^{\circ} \mathbf{C}\right]$ \\
\hline A & $68.0 \pm 0.0$ & $64.2 \pm 0.1$ \\
\hline Glycyl-L-asparagine & $68.1 \pm 0.2$ & $64.1 \pm 0.1$ \\
\hline L-arginine & $67.7 \pm 0.0$ & $63.8 \pm 0.0$ \\
\hline D(+)-trehalose & $67.7 \pm 0.0$ & $64.5 \pm 0.1$ \\
\hline None & $67.8 \pm 0.1$ & $64.4 \pm 0.2$ \\
\hline
\end{tabular}

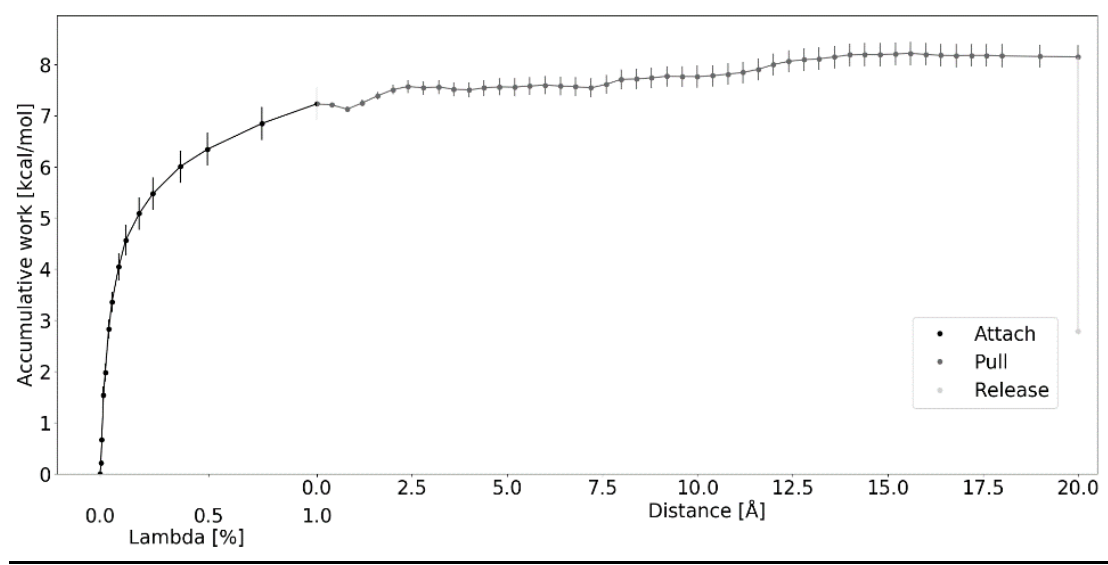

S-Figure 3: Potential of mean force depicting the work required to attach the restraints, to pull the ligand from its binding site and to release the restraints. The distance for the binding site is set to 0 . Error bars represent the standard error of the mean. 